\title{
A survey on SNCF decision support system tools to supervise and to pilot train traffic in operation
}

\author{
D. Gauyacq \\ SNCF Direction de la Recherche et de la Technologie, \\ Unité de Recherche "Génie Décisionnel Appliqué”, Paris, France
}

\begin{abstract}
In recent years, the SNCF Research Department has developed five different tools to monitor production operations. This supervision has the potential to affect the whole production process. This article will present the role played by the supervisory function in the production process and explain how this function contributes towards making the production process more efficient. In the second part, we shall be describing the basic principles of the tools used for supervisory purposes, both as regards the information systems that support them, the alarm, browse and display functions used for the production data, and the functions supporting the decision-making process. In the rest of the text, we shall be illustrating these principles by providing a brief description of two of the specific tools in use: GEDEON, for freight operations and EXCALIBUR, for overall management of trains in circulation. In conclusion, we shall attempt to show the work necessary to make these tools more efficient, whilst recognising that they can only be properly effective if associated with new forms of production organisation better adapted to the target purpose.
\end{abstract}

Keywords: supervision, operational management of traffic, man and machine interfaces, optimisation, quality of service, production system.

\section{Introduction}

Compared to most of the other existing transport modes, the railway can boast a large number of intrinsic advantages in terms of sustainable development. This is particularly true in the case of long-distance bulk traffic, an area where the energy required per unit carried is at its minimum. Yet, in a competitive 
environment with a market economy, this factor alone is not enough to make rail the obvious choice. In Europe, to enhance the future prospects of the rail mode, freight in particular, it is first and foremost necessary to boost production system efficiency. For this, substantial and quantifiable progress needs to be made to drive production costs radically down and vastly improve customer service standards. It is reasonable to conjecture that the action to be taken on these two fronts will be more complementary than contradictory, as we shall attempt to demonstrate in section 2.2.

This article will have the following structures: in the first part, we shall run briefly through the role played by the supervisory function in the production process. We shall be explaining how this function contributes towards making the production process more efficient. In the second part, we shall be describing the basic principles of the tools used for supervisory purposes, both as regards the information systems that support them, the alarm, browse and display functions used for the production data, and the functions supporting the decisionmaking process. In the rest of the text, we shall be illustrating these principles by providing a brief description of two of the specific tools in use: GEDEON, for freight operations and EXCALIBUR, for overall management of trains in circulation. In conclusion, we shall attempt to show the work necessary to make these tools more efficient, whilst recognising that they can only be properly effective if associated with new forms of production organisation better adapted to the target purpose.

\section{Supervision of production: a key factor in the industrialisation process}

\subsection{Role assigned to supervision}

The functional objectives associated with rail system production control are relatively easy to list, since the process consists of: (i) monitoring the execution of a production plan; (ii) checking that it is properly performed; (iii) reporting any disparities noted to an operator. Then he must, where relevant, anticipate other knock-on effects, in order to contain the consequences on the value of the service produced, in both quality and quantity terms. This process is in no way automatic, for the operator is positioned at the critical junction between observation of a problem and remedial action. (Actually, he acts in accordance with the principle of limited rationality as defined by H. Simon [5].) In the action he takes, the supervisor will use his professional experience, his knowledge of how the production process works and the margins for manoeuvre offered him by the system. This makes him a crucial player in the production process. (This need for a relatively impartial system of monitoring is virtually synonymous with the process of industrialisation. A good illustration of this is the "silent controller" introduced by Robert Owen [4] in the early $19^{\text {th }}$ century at his New Larnak factory. That innovation was theorised by Jeremy Bentham [1]. It is this same idea that was developed by Japanese engineer Taiichi Ohno (Toyota 
adviser) for whom "visual management" was vital [2].) It also means that he, too, needs to be supervised as part of the production process.

\subsection{Contribution of supervision to improving railway operating efficiency}

The rail network is a system based on economies of scale. (But is also a system of "economy of system" where productivity can be considerably enhanced without major investment but by improving the operational control/command system, primarily in order to co-ordinate the interaction between sub-systems and production players [3].) To drive down costs, it is therefore first necessary to make maximum use of railway infrastructure to reduce the unit operating cost, which in one way or another is always ultimately charged to production. Next, it is necessary to enhance the productivity of the main components of the production process, in other words the locomotives, wagons or coaches and workforce (including drivers). In fact, to exert proper control over production operations and thereby guarantee efficiency and quality, it is necessary to produce a formal definition of these operations. This definition, and above all its application, should not be allowed to have a diminishing effect or make it impossible for workers to take the initiatives necessary to meet the fundamental quality goals. It goes without saying that these different methods for enhancing system efficiency are mutually interdependent and part of an interrelated virtuous circle. Progress made in terms of lower costs and /or higher quality is bound to boost demand and thereby drive down fixed unit costs. Properly controlled and optimised technical production operations strictly applied should reduce the number of production incidents. In return this will make it possible to make greater use of production resources by lessening the unproductive margins set aside to allow for unexpected events, themselves often the direct result of improvised adaptation.

\section{Basic principle behind the supervisory tools developed at SNCF}

In recent years, the SNCF Research Department has developed five different tools to monitor production operations. This supervision has the potential to affect the whole of the production process: from the formation of trains in stations (AGORA), operation of TGV high-speed trains (COLT), suburban trains (ECLER), freight trains (GEDEON) but also to management at national level by the infrastructure manager of all these different movements (EXCALIBUR). The principles behind these tools are virtually identical in each case. Figure 1, shows in diagrammatic form the functional architecture we shall be describing in more precisely.

The main function of these tools is to monitor the actual implementation of the production plans and alert the operators as soon as any notable slippages appear. 
Each is based on four categories of information:

1) The production plan to be implemented, for example, the timetable diagram showing the trains to be worked, the routes they are to follow and the time at which they are expected to pass certain specific points;

2) The resources allocated to achieve this production plan: locomotives and driver rosters and, in addition, details of stopping (at platforms) and side-tracking;

3) Monitoring the production process via data showing, for example, the progress of trains over the network. This information comes mainly from fixed sensors installed in the track or from systems on board the vehicles themselves which enable their position to be established by GPS and transmitted by GSM;

4) The rules whereby it is possible to detect any slippage in the timing of the different operations in the production process. This can be seen or deduced, for example, when information expected fails to arrive.

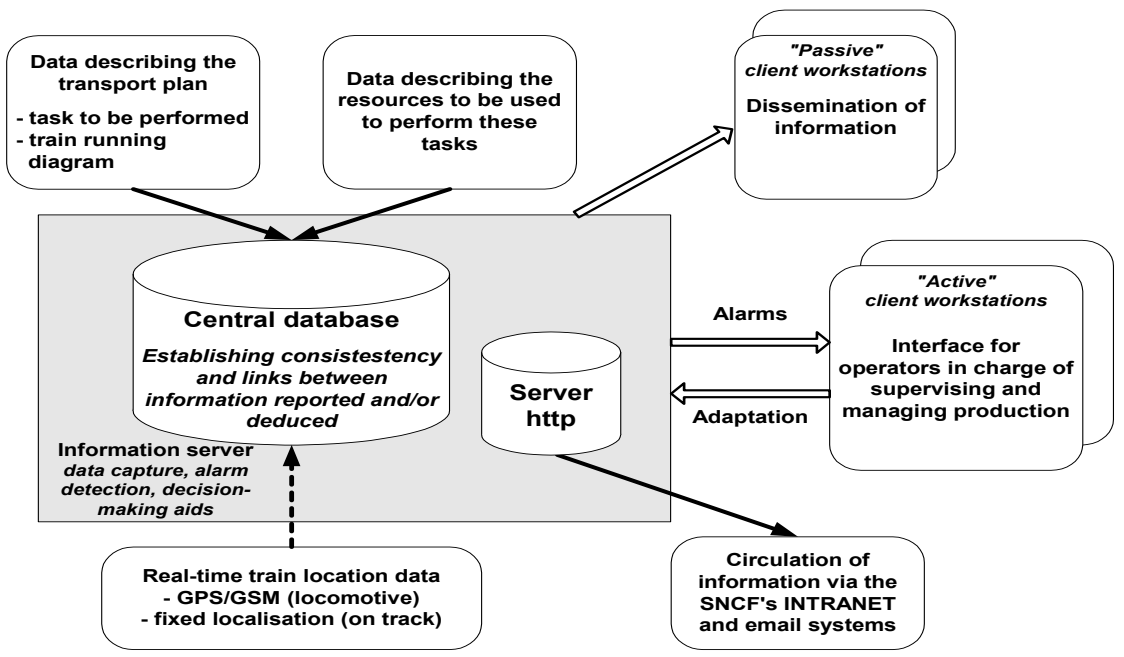

Figure 1.

All these tools are fed by information systems that existed before to their creation. In particular, we exploit the systems used to produce the different production plans for the specific types of traffic (passengers, freight, etc.) and/or specific resources (locomotives, driver, etc.). All information is periodically collected, generally on a daily basis, and input into a central database specific to each tool. Technically, all these tools are structured on a client/server basis so that data can be managed centrally and connected up to a large number of client (operator) workstations spread over a wide area, depending on the production requirements and organisation adopted. For a given application, this means distributing different services and information to the workstations connected to the network in relation to the different roles and functions of the operators working on them. 
To help operator in his task, the tool reports those production slippages that have reached a given threshold $\alpha_{i}$ in relation to category $i$ of the operation monitored, for example, when a train is still not reported as having passed a particular point $\alpha_{\text {train.delay }}$ minutes after the expected time. This threshold value is a parameter that the operator can adapt to circumstances. To make it easier for the operator to understand the problems, ergonomic interfaces display the potential consequences of the initial problem. For example, if a train is running late, this could mean that a driver travelling to work on this train would arrive late to take up his duty on train $T_{\mathbf{x}}$. The operator will have to work out a proposal to cater to this eventuality, for example, by switching round two driver rosters or by calling in a reserve driver or by noting the delay and the way it will affect train $T_{\mathbf{x}}$. In this last case, he should warn the department in charge of operating train $T_{x}$ to prevent it from being taken by surprise and, if necessary, to enable it to advise customers of the problem. To report problems encountered and decisions made, most of these tools have an http server connected to their database. This contains all the information judged useful in HTML format, which can then be polled via the SNCF's Intranet network. Access to this information is restricted to authorised personnel only.

The man/machine interfaces rely essentially on:

- Mapping data, in the form of geographical information systems displaying the physical location the different trains, locomotives, groups of wagons or the location of operating incidents;

- Graphic diagrams of the "time/resource" variety, for example, the rosters of the different locomotives in use. These show the resources used to complete a production task and the next tasks for which these resources are scheduled.

- Tables listing the alerts given, describing the delays incurred, the resources affected and the possible knock-on effects on other tasks;

- More detailed descriptions of the operations to be conducted, especially as regards train routes, schedules, driver's relief or locomotive unit transfer points, train composition, etc.

- Menus and/or tab bars to browse through pre-set screens, each of which may contain specific display and/or data, capture menus.

During the development of these tools, a number of decision-making aid functions were produced that have yet to be fully agreed by users. As a result, these functions are still at the prototype stage and require further enhancement. These support functions relate to three major categories of action which correspond, more or less, to the seriousness of the problem to be addressed and the time it will take for the situation to return more or less to normal:

- If/what tests to establish, more or less accurately, what the ultimate consequences of a particular decision will be on action in progress;

- Assessment and/or application of predetermined problem-solving scenarios. The operator may perform these actions automatically.

- Computing functions of the "operational research" type, to adapt the production schedule. 


\section{GEDEON - FREIGHT production supervision tool}

The tools (COLT, ECLER, GEDEON) used to supervise train movements are all based on similar functions. The differences between them lie essentially in the information systems to which they are connected. The most important of these differences relate to the type of traffic under control; other, more minor differences result from the way in which the production service in charge of the traffic is organised. In this paper, we shall simply describe GEDEON, since is widely used in the different FREIGHT production centres.

\subsection{GEDEON functions}

The central system supervises all FREIGHT sector trains on a real-time basis. Passenger trains headed by locomotives next scheduled to haul a FREIGHT train are also monitored. The corresponding information is placed at the disposal of the different production supervision centres. Each dedicated centre is considered to be a client of the central system and, as such, has the authority to supervise a series of trains. These latter are selected in line with the FREIGHT operations organisation which, from a supervisory standpoint, may (but does not necessarily always) have three categories of centre dedicated to:

a a type of traffic, for example, combined transport;

a major traffic route, in a bid to maximise flows;

a local zone in charge of gathering and delivering wagons.

GEDEON is supplied with the following information:

1. The data corresponding to the production schedule for a given period, for example 24 hours:

a The diagram of all FREIGHT train movements, some 2,500 trains each day;

- The list of wagon groups to be forwarded in these trains;

2. Data on the resources, locomotives and drivers earmarked for use in this production process:

a The references of the locomotives scheduled to haul the trains;

a The daily rosters of the drivers;

3. Production monitoring data assimilated to the pattern of train movements over the network:

- Information about the time trains are scheduled to pass specific points on the network captured via fixed transponders or input by signalmen;

- Automatic locomotive positioning data via GPS/GSM;

When a locomotive or a train is discovered to be running late by a given threshold delay, it is highlighted on the map at its latest known position and flagged up in the alarm tables. If the delay is unlikely to have any effect on the deployment of resources, a yellow symbol is used, otherwise the symbol is red. The resources (locomotive, driver) affected by this delay are also marked with the same colour code plus an indication of the estimated duration of the delay. 


\subsection{Illustration of man/machine interfaces}

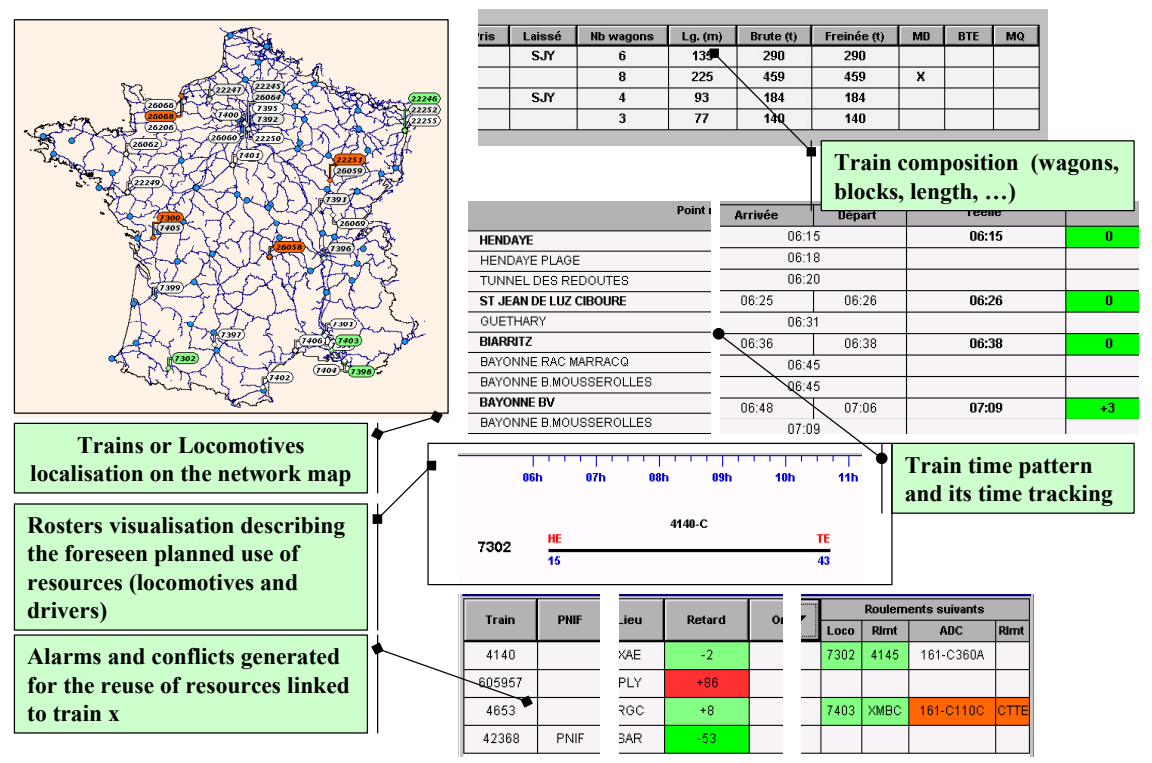

Figure 2: $\quad$ MMI to present information about freight traffic and resources of production.

\section{EXCALIBUR - overall train movement supervision tool}

EXCALIBUR is a tool that is in use at the SNCF's National Operations Centre (CNO). This centre is responsible for co-ordinating the activities of the 22 regional traffic control centres in charge of managing traffic for the tasks assigned to the infrastructure manager (IM). It is also the premise where the IM and the passenger and freight traffic operators can interact operationally to ensure that the knock-on effects of operating incidents affecting network capacity are managed with maximum efficiency.

\subsection{EXCALBUR functions}

The EXCALIBUR system is based on data describing the railway system. This description is geographically referenced, in other words the information may be shown on a skeleton map. The basic information comes from the RESEAU database and is used to attribute different qualities to the railway lines represented. As an example, the following line characteristics may be displayed on request: \{type of electrification; zone with/without traffic control; train running supervision system; possible throughput in relation to incidents reported; maximum speed; radio equipment; type of line (high-speed, conventional, narrow gauge); type of block section; operating system; type of traffic for each category (TGV, freight, main-line, Paris suburban)\}. The map also shows the national and SNCF administrative boundaries of the territory. It also shows the 
network in different degrees of detail depending on the amount of zoom applied. The most critical points in operating terms (tunnels, level crossings) can also be displayed, as can technical documents showing the track diagram of areas with complex layouts.

Any current incidents jeopardising the flow of traffic are marked on the map using symbols to show the degree to which throughput is affected. All the information available to describe the incident (time, place, cause, and consequences) can be accessed via the map references. Information about the development of the incident, action taken, steps to enable operations to continue are described, thus enabling each incident and its consequences to be traced and recorded. This adds up to an invaluable source of information and feedback for calculating the expected duration of future incidents and dynamically adapting these calculations to typical incident management techniques. For example, the knock-on effects of accidents involving people are always serious but the duration of the resulting traffic stoppage can vary widely, from one to several hours. It will be possible to give a more precise idea about the duration of each specific incident from information feedback by the system. This information may indicate whether or not the accident victim is still alive, whether or not the emergency services have reached the scene, whether or not the police or the judiciary officials are involved, etc.

The HOUAT system supplies details of the trains due to operate as part of the day's production schedule. The position data that can be used to follow train movements over the network is transmitted in real time by the BREHAT system. This information is supplemented by data input by EXCALIBUR users, who enter details of each incident, its repercussions and its development. This information is accessible to a whole series of duly authorised persons via Intranet (some 300 regularly connected users, when they need it). When an incident looks like affecting a region not immediately concerned, an email is sent to the traffic controllers in this region advising them to go to the EXCALIBUR site to follow developments.

For the moment EXCALIBUR offers two types of decision-making aid. The first is based on statistics approach and is designed to establish the likely duration of a given type of incident, the standard deviation for the particular incident type. This value is adjusted in relation to information coming in about the actual incident and its progression, action taken and the results observed. Work is at present in hand to introduce this approach on a systematic basis by using historical data on fixed installation failures and by finding the best way of grouping together and segmenting this data to establish the most accurate and reliable forecasts. The second relates to managing re-routing operations when major incidents prevent trains from running on certain sections of line. For the moment (cf. illustration) the support provided is more than information enabling the operator to:

a identify the trains to be re-routed and those already scheduled to run over alternative routes;

口 identify the cost of using a particular alternative route (extension of journey time, length, etc.); 
- select the right way to manage the incident (cancelling a train, maintaining it on the original route, diverting it, etc.

Ultimately this information will be extended to include action proposals suggested by an optimisation module that will (re)schedule current and future trains by attributing train paths and routes to minimise the consequences of the particular incident.

\subsection{Illustration through Man Machine Interfaces (MMI)}

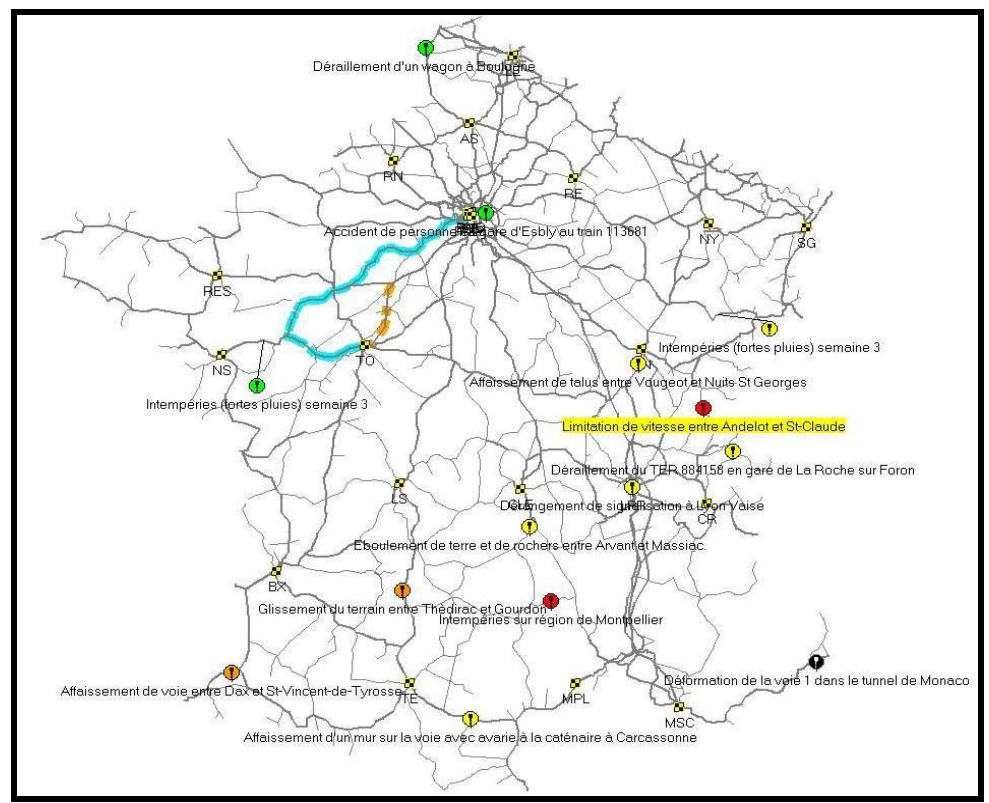

Figure 3: $\quad$ MMI to visualise infrastructure problems (colour circle) and rerouting possibilities (blue line) when a section line is blocked (dash yellow line).

\section{Conclusion and prospects}

The supervision tools presented above form sound foundations on which new projects in two complementary directions will be developed. The first of these involves improving production standards by tightening controls and attaching importance to performances achieved. Obviously, this can only be done if the production system is first standardised (as in the AGORA project) and by monitoring the action of those involved in the production process. (AGORA serves to supervise $(i)$ the preparation of trains and (ii) the flow of passengers alighting from arriving or boarding departing trains. Its main role is to ensure that the instructions contained in the quality manual are applied in practice. For example, a train (in its correct formation, cleaned, etc.) should be at the platform $\alpha, \mathbf{z}$ minutes before its departure time $\mathbf{t}_{\mathbf{0}}$; that the driver should report for duty on 
the train $\mathbf{x}$ minutes before $\mathbf{t}_{\mathbf{0}}$, and so on.) Where this latter point is concerned, the performance assessments (differences in relation to standards set and production results) allowed by these tools could be carried out for each of the production control centres, with the result that this form of accountability could play a dual role:

a checking that the various tasks are performed in line with the official procedures, that remedial action (with the help of tools in the case of complex procedures) is taken sufficiently quickly and thoroughly;

- Appreciation of the work done and the contribution of the different teams and individuals to ensuring that the overall system functions smoothly.

It goes without saying that this type of action should be part of a renewal of organisational and managerial system for it to be fully effective.

The second line of approach is more technical and would consist of introducing more efficient decision-making functionalities:

- to be able better to anticipate the consequences of operating problems and incidents and provide information facilitating the co-ordination of the production players and, by extension, the customer (by making the first such functions systematic in EXCALIBUR);

- to adapt the production plans as they are being carried out on a semiautomatic basis when, for whatever reason, it is no longer possible to stick to the original plan.

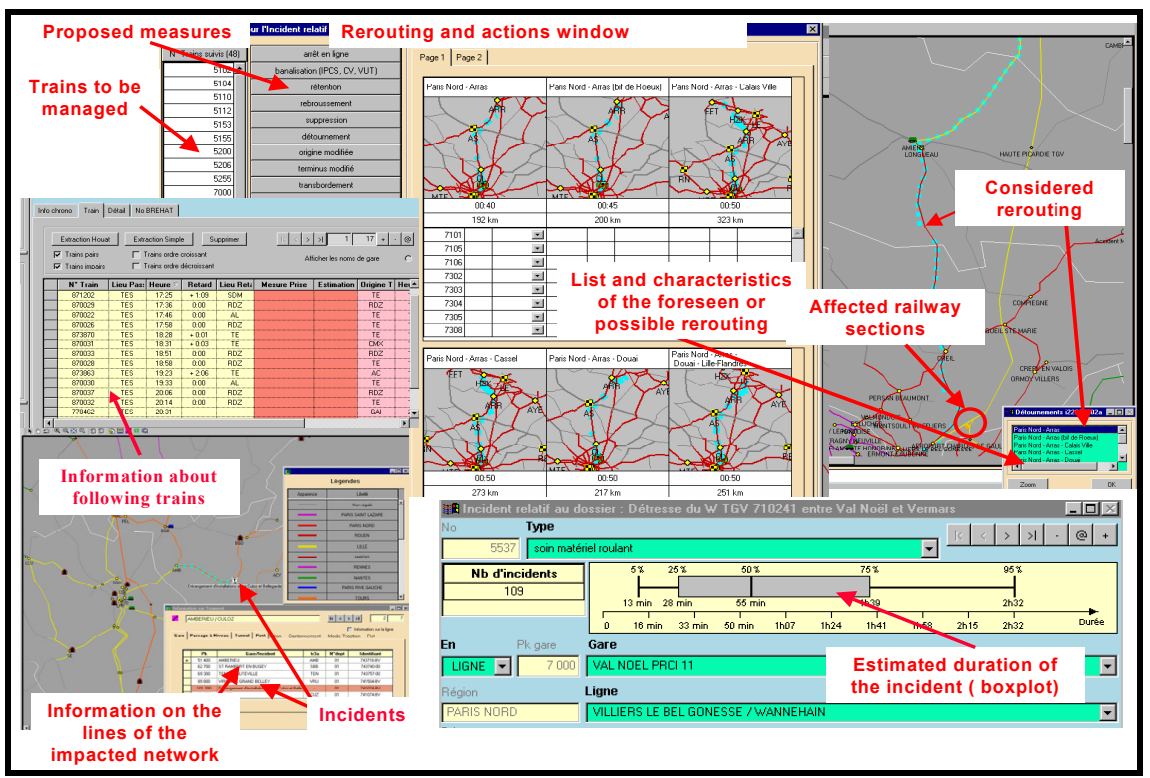

Figure 4: $\quad$ MMI to manage rerouting of trains when a line section is blocked.

In both cases, the extra functions that need to be developed or enhanced can only provide support and assistance in actions that involve changing (or at least stimulating and boosting) the existing production organisation. 
In our opinion, the main contribution of these tools and the steps planned to build up and extend their supervisory functions lies in making staff recognise the need for change and motivating them to improve their production standards. The knock-on effect is to enhance productivity through tighter control of operations. Conversely, for these tools to work efficiently they need to be used by staff with the requisite new skills and, above all, the ability to understand the problemsolving logic involved so they can properly interpret and exploit their new resources.

\section{References}

[1] Jeremy Bentham Optique The Panopticon Writings, Edition Mille et Une Nuits, No. 398, 2002.

[2] Benjamin Coriat, "Penser à l'envers, Travail et organisation dans l'Entreprise japonaise" (Thinking backwards and Japanese company organisation), Christian Bourgeois Publisher, 1991.

[3] Bernard Lacroix Technical Systems approach applied to the Air Traffic Management: the central role of the economic forces", Conference Delft, 2001, www.delft2001.tudelft.nl/paper\%20files/paper1128.doc

[4] R. Owen, "Textes choisis" (Selected texts), p 79, Editions Sociales, 1963

[5] H.A. Simon, J.-G. Marches organisations" (Organisations), DUNOD, 1999. 